\title{
Interrelationships among Whole-Body Coordination, Functional Potentiality, and Environmental Adaptability
}

\author{
Katsuyasu Kouda \\ Department of Hygiene, Kansai Medical University
}

\begin{abstract}
The term "adaptability" or "capacity of adaptation" is the central concept in the general advancement and promotion of research in physiological anthropology. Throughout the history of Homo sapiens, mankind has adapted itself to environmental stress. As a result, numerous physiological polymorphisms in humans are present in our planet-wide distribution. Totally regulated physiological function by integration and coordination is referred to as whole-body coordination and is associated with a high degree of adaptability in humans. Functional potentiality also affects environmental adaptability. Thus, whole-body coordination and functional potentiality are necessary for adaptation to environmental changes. There is an interrelationship among functional potentiality, whole-body coordination, physiological polymorphisms, and environmental adaptability. J Physiol Anthropol Appl Human Sci 24(3): 241-242, 2005 http:// www.jstage.jst.go.jp/browse/jpa
\end{abstract}

[DOI: 10.2114/jpa.24.241]

keywords: adaptation, capacity, environment, evolution, human

\section{High Capacity for Human Adaptation}

Humans are a species with a highly developed capacity for adaptation to environmental stress. Our planet-wide distribution and survival in diverse and stressful habitats is a result of our high degree of environmental adaptability. Over the long period of time from the first appearance of humans, frequent adaptations have occurred to allow survival in the changing environment. People have now adapted to the total range of terrestrial environments and have spread beyond their original habitats (Harrison et al., 1988; Huss-Ashmore, 2000).

\section{Adaptability}

The term "adaptability" or "capacity of adaptation" is the central concept in the general advancement and promotion of research in physiological anthropology. Adaptation may be classified as genetic, physiological, developmental, and behavioral adaptive responses in the research field of human biology (Harrison et al., 1988; Huss-Ashmore, 2000). In contrast, environmental adaptability may be defined as "physiological environmental adaptability" in the research field of physiological anthropology.

\section{Polymorphism and Adaptability}

During the history of Homo sapiens, mankind has adapted itself to environmental stresses such as heat, cold, ultraviolet radiation, hypoxia, bacteria, viruses, worms, poisons, allergens, too few nutrients, and an overabundance of nutrients. Thus, various environmental factors have played an important role in our evolution. As a result, humans have acquired numerous variations in both genotype and phenotype. Different human populations show different responses to the same stressors. Furthermore, evolution continues to be ongoing in highly developed technological societies.

Adaptation, chance, and history have all played a role in producing physiological polymorphism, which consists of our biological diversity (Harrison et al., 1988; Huss-Ashmore, 2000). Thus, numerous physiological polymorphisms in humans are also the result of the high degree of environmental adaptability in humans.

\section{Homeostasis}

Cannon coined the term "homeostasis" to describe "the various physiologic arrangements which serve to the normal state, once it has been disturbed" (Cannon, 1932). A large part of physiology is therefore concerned with regulatory mechanisms that act to maintain the constancy of the internal environment (Ganong, 2001).

\section{Homeostasis and Coordination}

The nervous system, the senses, the endocrine system, and 


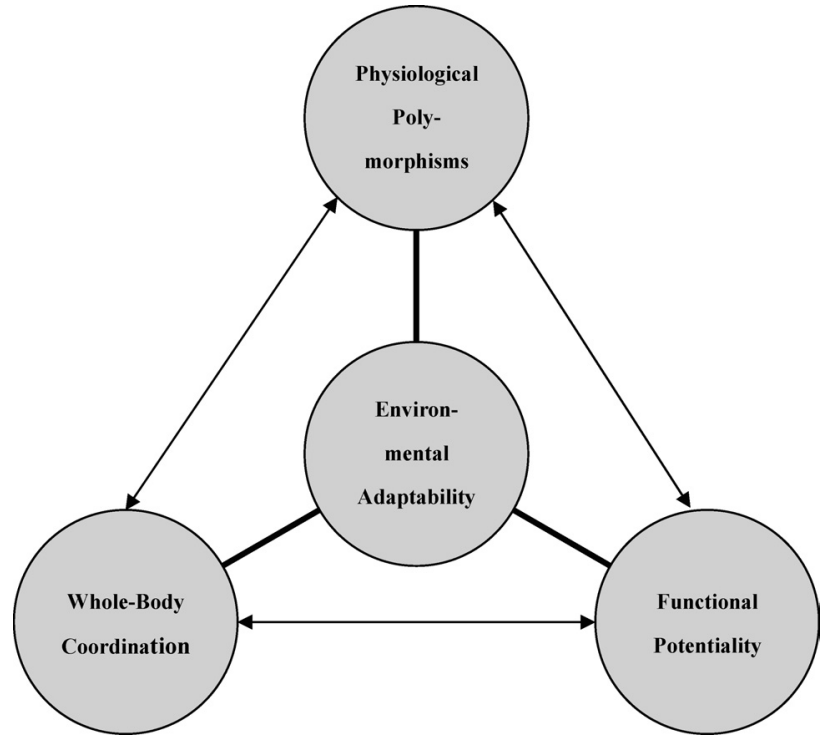

Fig. 1 Interrelationships among functional potentiality, whole-body coordination, physiological polymorphisms, and environmental adaptability.

other chemical signals regulate organs in humans. These regulating systems do not work individually. Integration and coordination of these systems (Mader, 2004) are necessarily to maintain homeostasis.

Totally regulated physiological function by means of integration and coordination is referred to as whole-body coordination. Whole-body coordination consists of not only coordination among the nervous system, the senses, the endocrine system, and other chemical signals, but also coordination among the functions of all parts of the body, such as bone, muscles, heart, vessels, blood, lungs, gut, liver, gallbladder, pancreas, kidneys, and genitalia.

A high degree of competence in whole-body coordination results in a high degree of adaptability. There is a positive relationship between whole-body coordination and adaptability. Therefore, the physiological anthropologist is interested in this term.

\section{Homeostasis and Potentiality}

Manifestations of new physiological functions are shown in embryonic development, fetal development, and the development of male and female sex organs. After birth, manifestations of new physiological functions and changes in physiological function also continue during infancy, childhood, puberty, and adolescence. During the growth periods, manifestations of new physiological functions are affected by changes in environment, experience, education, training, and age.

Occasionally, environments expose humans to severe stress, threatening to disrupt normal homeostasis. In times of dangerous stress, some of the latent ability of physiological function appears in the nervous system, endocrine system, chemical signals, and organs, thus maintaining homeostasis in the living body. A high degree of competence in the latent ability of physiological functions enables an organism to survive during dangerous environmental change. Therefore, there is a positive relationship between functional potentiality and adaptability. Functional potentiality also affects environmental adaptability.

\section{Conclusion}

Whole-body coordination and functional potentiality are necessary for adaptation to environmental changes. Therefore, both whole-body coordination and functional potentiality are strongly associated with environmental adaptability.

Acknowledgments This work was supported in part by a Grant-in-Aid for Scientific Research (B) of the Japan Society for the Promotion of Science (15370102).

\section{References}

Cannon WB (1932) The wisdom of the body. New York, Norton

Ganong WF (2001) Review of medical physiology. 20th ed., McGraw-Hill, New York, 46

Harrison GA, Tanner JM, Pilbeam DR, Baker PT (1988) Human biology: an introduction to human evolution, variation, growth, and adaptability. 3rd ed., Oxford University Press, Oxford, 439-449

Huss-Ashmore R (2000) Theory in human biology: evolution, ecology, adaptability, and variation. In Stinson S, Bogin B, Huss-Ashmore R, O'Rourke D eds., Human biology: an evolutionary and biocultural perspective. Wiley-Liss, New York, $1-25$

Mader SS (2004) Human biology. 8th ed., McGraw-Hill, New York, 221-292

Received: December 13, 2004

Accepted: March 9, 2005

Correspondence to: Katsuyasu Kouda, MD, PhD., Department of Hygiene, Kansai Medical University, 10-15 Fumizono-cho, Moriguchi, Osaka 570-8506, Japan

Phone: +81-6-6993-9436

Fax: +81-6-6992-3522

e-mail:koudak@takii.kmu.ac.jp 